\title{
Improved Non-committing Encryption with Applications to Adaptively Secure Protocols
}

\author{
Seung Geol Choi ${ }^{1, \star}$, Dana Dachman-Soled ${ }^{1, \star}$, Tal Malkin ${ }^{1, \star}$, and Hoeteck Wee ${ }^{2, \star \star}$ \\ 1 Columbia University \\ \{sgchoi, dglasner, tal\}@cs. columbia.edu \\ 2 Queens College, CUNY \\ hoeteck@cs . qc . cuny . edu
}

\begin{abstract}
We present a new construction of non-committing encryption schemes. Unlike the previous constructions of Canetti et al. (STOC '96) and of Damgård and Nielsen (Crypto '00), our construction achieves all of the following properties:
\end{abstract}

- Optimal round complexity. Our encryption scheme is a 2-round protocol, matching the round complexity of Canetti et al. and improving upon that in Damgård and Nielsen.

- Weaker assumptions. Our construction is based on trapdoor simulatable cryptosystems, a new primitive that we introduce as a relaxation of those used in previous works. We also show how to realize this primitive based on hardness of factoring.

- Improved efficiency. The amortized complexity of encrypting a single bit is $O(1)$ public key operations on a constant-sized plaintext in the underlying cryptosystem.

As a result, we obtain the first non-committing public-key encryption schemes under hardness of factoring and worst-case lattice assumptions; previously, such schemes were only known under the CDH and RSA assumptions. Combined with existing work on secure multi-party computation, we obtain protocols for multi-party computation secure against a malicious adversary that may adaptively corrupt an arbitrary number of parties under weaker assumptions than were previously known. Specifically, we obtain the first adaptively secure multi-party protocols based on hardness of factoring in both the stand-alone setting and the UC setting with a common reference string.

Keywords: public-key encryption, adaptive corruption, non-committing encryption, secure multi-party computation.

\section{Introduction}

Secure multi-party computation (MPC) allows several mutually distrustful parties to perform a joint computation without compromising, to the greatest extent possible,

\footnotetext{
* Supported in part by NSF Grants CCF-0347839, CNS-0716245, CNS-0831094 and SBE0245014.

${ }^{\star \star}$ Partially supported by a PSC-CUNY Award, and part of this work was done while a post-doc at Columbia University.
} 
the privacy of their inputs or the correctness of the outputs. An important criterion in evaluating the security guarantee is how many parties an adversary is allowed to corrupt and when the adversary determines which parties to corrupt. Ideally, we want to achieve the strongest notion of security, namely, against an adversary that corrupts an arbitrary number of parties, and adaptively determines who and when to corrupt during the course of the computation (and without assuming erasures). Even though the latter is a very natural and realistic assumption about the adversary, most of the MPC literature only addresses security against a static adversary, namely one that chooses (and fixes) which parties to corrupt before the protocol starts executing. And if indeed such protocols do exist, it is important to answer the following question:

What are the cryptographic assumptions under which we can realize MPC protocols secure against a malicious, adaptive adversary that may corrupt a majority of the parties?

Towards answering this question, we revisit the problem of constructing noncommitting encryption schemes, a cryptographic primitive first introduced by Canetti et al. [CFGN96] as a tool for building adaptively secure MPC protocols in the presence of an honest majority. Informally, non-committing encryption schemes are semantically secure, possibly interactive encryption schemes, with the additional property that a simulator can generate special ciphertexts that can be opened to both a 0 and a 1 . In a more recent work, Canetti et al. [CLOS02] (extending [B98]) showed how to construct adaptively secure oblivious transfer protocols starting from non-committing public-key encryption schemes (i.e. the key generation algorithm must be non-interactive), which may in turn be used to construct MPC protocols secure against a malicious, adaptive adversary that may corrupt an arbitrary number of parties.

Unfortunately, the only known constructions of non-committing public-key encryption schemes (PKEs) are based on the CDH and RSA assumptions [CFGN96] and the construction exploits in a very essential way that these assumptions give rise to families of trapdoor permutations with a common domain. If we allow for an interactive key generation phase, Damgård and Nielsen [DN00], building on [B97, CFGN96], constructed 3-round non-committing encryption schemes based on a more general assumption, that of simulatable PKEs, which may in turn be realized from DDH, CDH, RSA and more recently, worst-case lattice assumptions [GPV08] (see figure 1).

\subsection{Our Results}

First, we present a new construction of non-committing encryption schemes, which simultaneously improves upon all of the previous constructions in [CFGN96, DN00:

Optimal Round Complexity. We provide a construction of non-committing PKEs from simulatable cryptosystems. Our construction is surprisingly simple - a twist to the standard cut-and-choose techniques used in [DN00, KO04] - and also admits a fairly

\footnotetext{
${ }^{1}$ Refer to [C00, Section 5.2] for a discussion on how trusted erasures may be a problematic assumption.
} 
straight-forward simulation and analysis. In particular, our construction and the analysis are conceptually and technically simpler than those in [CFGN96, DN00]; we avoid having to analyze the number of one's in certain Binomial distributions as in [CFGN96] and to consider a subtle failure mode as in [DN00].

Reducing the assumptions. Informally, a simulatable PKE is an encryption scheme with special algorithms for obliviously sampling public keys and random ciphertexts without learning the corresponding secret keys and plaintexts; in addition, both of these oblivious sampling algorithms should be efficiently invertible.

We define a weaker assumption, which we refer to as trapdoor simulatable cryptosystems, and prove that it is sufficient for our construction and analysis to go through. Roughly speaking, we provide the inverting algorithms in a simulatable cryptosystem with additional trapdoor information (hence the modifier "trapdoor"), which makes it easier to design a simulatable cryptosystem.

Improved efficiency. While the main focus of this work is feasibility results (notably, reducing the computational assumptions for both non-committing encryption schemes and adaptively secure MPC), we show how to combine a variant of our basic construction with the use of error-correcting codes to achieve better efficiency. That is, the amortized complexity of encrypting a single bit is $O(1)$ public-key operations on a constant-sized plaintext in the underlying cryptosystem.

Thus, we obtain the following.

Theorem 1 (informal). There exists a black-box construction of a non-committing public-key encryption scheme, starting from any trapdoor simulatable cryptosystem.

Factoring-Based constructions. Next, we derive trapdoor simulatable cryptosystems from a variant of Rabin's trapdoor permutations (c.f. [H99, S96, FF02]) based on the hardness of factoring Blum integers.

Theorem 2 (informal). Suppose factoring Blum integers is hard on average. Then, there exists a trapdoor simulatable cryptosystem.

We stress that we do not know how to construct a simulatable cryptosystem under the same assumptions; specifically, inverting the sampling algorithm for ciphertexts in our construction without the trapdoor (the factorization of the Blum integer modulus) appears to be as hard as factoring Blum integers. This shows that trapdoor simulatable cryptosystems is indeed a meaningful and useful relaxation. In the process, we also obtain the first factoring-based dense cryptosystems 2 When combined with enhanced trapdoor permutations, this yields the first factoring-based non-interactive proofs of knowledge [DP92].

Oblivious Transfer and MPC. We consider the applications of our main result to the constructions of adaptively secure oblivious transfer and general MPC protocols in both the stand-alone setting and the UC setting (c.f. [CLOS02, IPS08, CDSMW09]).

\footnotetext{
${ }^{2}$ These are PKE schemes where a random string has a inverse polynomial probability of being a valid public key.
} 


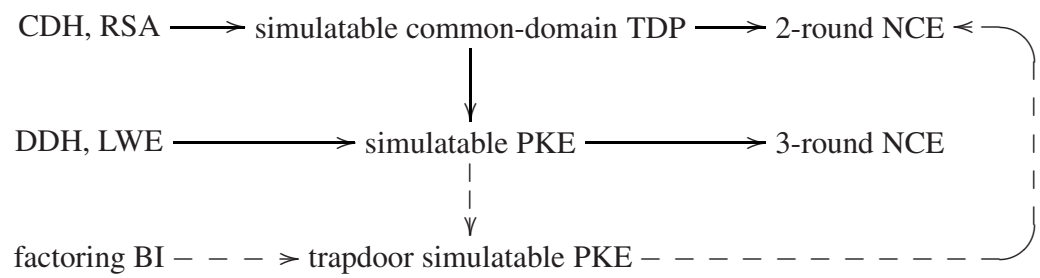

Fig. 1. Summary of previous results (solid lines) along with our contributions (dashed lines)

Theorem 3 (informal). There exists a black-box construction of a 6-round 1-out-of$\ell$ oblivious transfer protocol for strings in the $\mathcal{F}_{\mathrm{COM}}$-hybrid mode 3 in the UC setting that is secure against a malicious, adaptive adversary, starting from any trapdoor simulatable cryptosystem.

We add that if the oblivious key generation algorithm in the trapdoor simulatable cryptosystem achieves statistical indistinguishability (which is the case for all of the afore-mentioned constructions), then we obtain an OT protocol that is secure against a computationally unbounded malicious sender. While our OT protocol is not as efficient as that in the recent work of Garay, Wichs and Zhou [GWZ09] (we incur an additional multiplicative overhead that is linear in the security parameter), our protocol along with our general framework offers several advantages:

- In addition to relying on the $\mathcal{F}_{\mathrm{COM}}$ functionality and a simulatable PKE (to implement non-committing encryption) as in our work, the GWZ09 framework requires a so-called enhanced dual-mode cryptosystem. This is a relatively highlevel CRS-based primitive from [PVW08] augmented with two main additional properties: the first has a flavor of oblivious sampling; the second requires that the underlying CRS be a common random string (modulo some system parameters) and not just a common reference string. This requirement is inherent to their framework, since this CRS is generated using a coin-tossing protocol. This latter requirement is very restrictive, and the only known construction of an enhanced dual-mode cryptosystem is based on the quadratic residuocity assumption.

- Our protocol immediately handles 1-out-of- $\ell$ OT, whereas [GWZ09] only addresses 1-out-of-2 OT, a limitation inherited from [PVW08].

Combined with CLOS02, IPS08, CDSMW09], we obtain the following corollaries:

Corollary 1 (informal). Assuming the existence of trapdoor simulatable cryptosystems, there exists adaptively secure multi-party protocols in the stand-alone setting and in the $\mathcal{F}_{\mathrm{COM}}$-hybrid model in the UC setting against a malicious adversary that may adaptively corrupt any number of parties.

Specifically, we obtain the first adaptively secure multi-party protocols based on hardness of factoring in both the stand-alone setting and the UC setting with a common reference string.

\footnotetext{
${ }^{3} \mathcal{F}_{\text {COM }}$ is an ideal functionality for commitment.
} 


\subsection{Additional Related Work}

The problem of constructing encryption schemes that are secure against adaptive corruptions was first addressed in the work of Beaver and Haber [BH92]. They considered a simpler scenario where the honest parties have the ability to securely and completely erase previous states. For instance, an honest sender could erase the randomness used for encryption after sending the ciphertext, so that upon being corrupted, the adversary only gets to see the corresponding plaintext. An intermediate model, wherein we assume secure erasures for either the sender or receiver but not both (or, by limiting the adversary to corrupting at most one of the two parties), has been considered in several other works [JL00, CHK05, KO04].

Organization. We present an overview of our constructions in Section 2 preliminaries in Section 3 the formulation of a trapdoor simulatable PKE in Section 4, our factoringbased trapdoor simulatable PKE in Section 6, and our non-committing encryption scheme in Section 5 In Section 7, we show the construction of a 6-round oblivious transfer protocol.

\section{Overview of Our Constructions}

At a high level, our non-committing PKE is similar to that from previous works [CFGN96, DN00, KO04]. The receiver generates a collection of public keys in such a way that it only knows an $\alpha$ fraction of the corresponding secret keys; this can be achieved by generating an $\alpha$ fraction of the public keys using the key generation algorithm and the remaining $1-\alpha$ fraction obliviously. Similarly, the sender generates a collection of ciphertexts in such a way that it only knows an $\alpha$ fraction of the corresponding plaintexts. Previous constructions all work with the natural choice of $\alpha=1 / 2$ so that the simulator generates a collection of ciphertexts half of which are encryptions of 0 and the other half are encryptions of 1. As noted in [KO04, this is sufficient for obtaining non-committing PKEs wherein at most one party is corrupted. Roughly speaking, the difficulty in handling simultaneous corruptions of both the sender and the receiver with $\alpha=1 / 2$ is that in the simulation, the sender's choice of the $\alpha$ fraction of keys completely determine the receiver's choice of the $\alpha$ fraction of ciphertexts whereas in an actual honest encryption, these choices are completely independent (we elaborate on this later in this section). The key insight in our construction is to work with a smaller value of $\alpha$ (turns out $1 / 4$ is good enough).

A Toy Construction. Consider the following encryption scheme, which is a simplification of that in $\mathrm{KO} 04, \mathrm{DN} 00$. The receiver generates a pair of public keys $\left(\mathrm{PK}_{0}, \mathrm{PK}_{1}\right)$ by generating one key (selected at random) using the key-generation algorithm, and the other using the oblivious sampling algorithm. To encrypt a bit $b$, the sender generates a pair of ciphertexts $\left(C_{0}, C_{1}\right)$ as follows: pick a random bit $r$, set $C_{r}$ to be $\operatorname{Enc}_{\mathrm{PK}_{r}}(b)$ and choose $C_{1-r}$ using the oblivious sampling algorithm. To decrypt, the receiver decrypts exactly one of $C_{0}, C_{1}$ using the secret key that it knows. This construction corresponds to $\alpha=1 / 2$ where $\alpha$ is the fraction of public keys for which the receiver knows the secret key, and also the fraction of ciphertexts for which the sender knows the plaintext. Observe that this encryption scheme has the following properties: 
- It has a constant decryption error of $1 / 4$ if an obliviously sampled ciphertext is equally likely to decrypt to 0 or 1 . As shown in [KO04], this error can be reduced by standard repetition techniques.

- It tolerates corruption of either the sender or the receiver, but not both. Consider a simulator that generates both of $\left(\mathrm{PK}_{0}, \mathrm{PK}_{1}\right)$ (along with $\left.\mathrm{SK}_{0}, \mathrm{SK}_{1}\right)$ using the keygeneration algorithm, and a ciphertext $\left(C_{0}, C_{1}\right)$ as follows: pick a random bit $\beta$, and set $C_{0}$ to be $\operatorname{Enc}_{\mathrm{PK}_{0}}(\beta)$ and $C_{1}$ to be $\operatorname{Enc}_{\mathrm{PK}_{1}}(1-\beta)$. Suppose the simulator later learns that this is an encryption of 0 . If only the sender is corrupted, the simulator claims $r=\beta$ and that $C_{1-\beta}$ is obliviously sampled. If only the receiver

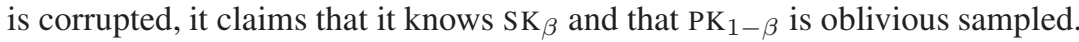

We highlight two subtleties in the above simulation strategy. First, it achieves 0 decryption error (as opposed to $1 / 4$ in an honest encryption); this can be fixed with a somewhat more involved simulation strategy. This in turn becomes pretty complicated once we use standard repetition techniques to reduce the decryption error. Next, it is always the case in the simulation that either both $\mathrm{PK}_{0}$ and $C_{0}$ are obliviously sampled, or both $\mathrm{PK}_{1}$ and $C_{1}$ are obliviously sampled. As such, this simulation strategy fails if both the sender and the receiver are corrupted, because in an actual encryption, which of $\mathrm{PK}_{0}, \mathrm{PK}_{1}$ and which of $C_{0}, C_{1}$ are obliviously sampled are determined independently.

Our Encryption Scheme. As noted in the introduction, the key insight in our construction is to work with a small value of $\alpha$. In addition, following [DN00], we use a random $k$-bit encoding of 0 and 1 , where $k$ is the security parameter:

- The receiver generates $4 k$ public keys $\mathrm{PK}_{1}, \ldots, \mathrm{PK}_{4 k}: k$ of them are generated using the key-generation algorithm, and the remaining $3 k$ are generated using the oblivious sampling algorithm. The receiver then sends $\mathrm{PK}_{1}, \ldots, \mathrm{PK}_{4 k}$ along with two random $k$-bit messages $M_{0}, M_{1}$.

- To encrypt a bit $b$, the sender sends $4 k$ ciphertexts (one for each of $\mathrm{PK}_{1}, \ldots, \mathrm{PK}_{4 k}$ ), of which $k$ are encryptions of $M_{b}$, and the remaining ones are obliviously sampled.

- To decrypt, the receiver decrypts the $k$ ciphertexts for which it knows the corresponding secret key. If any of the $k$ plaintexts matches $M_{0}$, it outputs 0 and otherwise, it outputs 1 .

Encoding 0 and 1 randomly as $M_{0}$ and $M_{1}$ is useful for two reasons:

- That an obliviously sampled ciphertext is equally likely to decrypt to 0 or 1 is no longer needed to guarantee correctness (c.f. [DN00]). Indeed, reasoning about decryptions of obliviously sampled ciphertext is non-trivial for the lattice-based simulatable PKEs in [GPV08].

- Constructing a simulator becomes much easier as we avoid having to generate distributions over $k$ independent biased bits conditioned on the majority of the bits being 0, say. Generating such distributions arises for instance in [CFGN96 and is related to the first subtlety associated with the naive simulation strategy. In our construction, the simulated ciphertext comprises $k$ encryptions of $M_{0}, k$ encryptions of $M_{1}$, and $2 k$ obliviously generated ciphertexts. Having these extra $2 k$ obliviously generated ciphertexts (which is possible because $\alpha<1 / 2$ ) is crucial for handling simultaneous corruptions of the sender and the receiver. 
Trapdoor Simulatable PKEs from Factoring. Our factoring-based trapdoor simulatable PKE construction consists of two main steps. First, we modify the Rabin trapdoor permutations based on squaring modulo Blum integer so that it remains a permutation over any arbitrary integer modulus. This relies on the following number-theoretical structural lemma implicit in [H99, S96, FF02]:

Let $N$ be an arbitrary odd $k$-bit integer, and let $Q_{N}=\left\{a^{2^{k}}(\bmod N) \mid a \in\right.$ $\left.Z_{N}^{*}\right\}$. Then, the map $\psi: x \mapsto x^{2}$ defines a permutation over $Q_{N}$.

We also provide an efficient algorithm for inverting $\psi$ given the factorization of $N$. Note that the standard algorithm for computing square roots does not guarantee that the output lies in $Q_{N}$. Moreover, the probability that a random square root lies in $Q_{N}$ may be exponential small so we cannot repeatedly computing random square roots until we find one in $Q_{N}$; it's also not clear a-priori how to test membership in $Q_{N}$ even given the factorization of $N$.

The next step transforms the family of trapdoor permutations $\psi$ acting on the domain $Q_{N}$ into a family of "enhanced" trapdoor permutations with the same domain $Q_{N}$, using an idea from [G04, Section C.1]. The latter has the property that we can obliviously sample a random element $y$ in $Q_{N}$ so that given $y$ along with the coin tosses used to sample $y$, it is infeasible to compute the preimage of $y$ under the permutation (note that the naive algorithm for sampling a random element of $Q_{N}$ gives away its preimage under $\psi$ ). We will need the oblivious sampling algorithm for a random element in $Q_{N}$ in our oblivious sampling algorithm for random ciphertexts. We will also need to realize trapdoor invertibility for the latter, which requires an efficient algorithm that given the factorization of $N$ and an element $y$ in $Q_{N}$, outputs a random $2^{k}$ 'th root of $y 5$ Note that iteratively computing random square roots $k$ times does not work: after computing the first square root, we may not end up with a $2^{k-1}$ 'th power.

\section{Preliminaries}

If $A$ is a probabilistic polynomial time (hereafter, ppt) algorithm that runs on input $x$, $A(x)$ denotes the random variable according to the distribution of the output of $A$ on input $x$. We denote by $A(x ; r)$ the output of $A$ on input $x$ and random coins $r$. To simplify the notation, we will often omit quantifying over the distribution for $r$; it will usually be clear from the context when $r$ is not fixed, that it is drawn from the uniform distribution over strings of the appropriate length.

We assume that the reader is familiar with the standard definitions of public-key encryption schemes and semantic security (c.f. [GM84, G04]). We stress that we allow decryption errors that are exponentially small in $k$ :

\footnotetext{
${ }^{4}$ It was shown in [H99] that $\psi$ defines a permutation over the subgroup $O_{N}$ of $Z_{N}^{*}$ of odd order, and that $O_{N}$ contains $Q_{N}$; turns out $O_{N}=Q_{N}$. While $Q_{N}$ is trivially sampleable, it is not clear a-priori how to sample from $O_{N}$.

${ }^{5}$ If we are given just $N$ and not its factorization, this problem is at least as hard as factoring random Blum integers. This is in essence why we only obtain a factoring-based trapdoor simulatable PKE and not a simulatable PKE.
} 
Definition 1 (encryption scheme). A triple (Gen, Enc, Dec) is an encryption scheme, if Gen and Enc are ppt algorithms and Dec is a deterministic polynomial-time algorithm such that for every message $m \in\{0,1\}^{*}$ of polynomial length, $\operatorname{Pr}\left[\operatorname{Gen}\left(1^{k}\right) \rightarrow\right.$ $\left.(\mathrm{PK}, \mathrm{SK}), \operatorname{Enc}_{\mathrm{PK}}(m) \rightarrow c ; \operatorname{Dec}_{\mathrm{SK}}(c) \neq m\right]<2^{-\Omega(k)}$.

Non-committing Encryption. For simplicity, we present the definition of a noncommitting public-key encryption scheme for single-bit messages:

Definition 2 (non-committing encryption [CFGN96). $A$ non-committing (bit) encryption scheme consists of a tuple (NCGen, NCEnc, NCDec, NCSim) where (NCGen, NCEnc, NCDec) is an encryption scheme and NCSim is the simulation algorithm that on input $1^{k}$, outputs $\left(e, c, \sigma_{\mathrm{G}}^{0}, \sigma_{\mathrm{E}}^{0}, \sigma_{\mathrm{G}}^{1}, \sigma_{\mathrm{E}}^{1}\right)$ with the following property: for $b=0,1$ the following distributions are computationally indistinguishable:

- the joint view of an honest sender and an honest receiver in a normal encryption of $b$ :

$$
\left\{\left(e, c, \sigma_{\mathrm{G}}, \sigma_{\mathrm{E}}\right) \mid(e, d)=\operatorname{NCGen}\left(1^{k} ; \sigma_{\mathrm{G}}\right), c=\operatorname{NCEnc}_{e}\left(b ; \sigma_{\mathrm{E}}\right)\right\}
$$

- simulated view of an encryption of $b$ :

$$
\left\{\left(e, c, \sigma_{\mathrm{G}}^{b}, \sigma_{\mathrm{E}}^{b}\right) \mid \operatorname{NCSim}\left(1^{k}\right) \rightarrow\left(e, c, \sigma_{\mathrm{G}}^{0}, \sigma_{\mathrm{E}}^{0}, \sigma_{\mathrm{G}}^{1}, \sigma_{\mathrm{E}}^{1}\right)\right\}
$$

It follows from the definition that a non-committing encryption scheme is also semantically secure.

Encrypting longer messages. Starting with a non-committing bit encryption scheme (NCGen, NCEnc, NCDec, NCSim), we may encrypt a longer message of length $n$ by generating $n$ independent public keys using NCGen, encrypting each bit of the message using a different public key and then concatenating the $n$ ciphertexts. Note that this is different from the case of semantically secure encryption, where we may encrypt each bit using the same public key.

\section{Trapdoor Simulatable Public Key Encryption}

A $\ell$-bit trapdoor simulatable encryption scheme consists of an encryption scheme (Gen, Enc, Dec) augmented with (oGen, oRndEnc, rGen, rRndEnc). Here, oGen and oRndEnc are the oblivious sampling algorithms for public keys and ciphertexts, and $r$ Gen and rRndEnc are the respective inverting algorithms. We require that, for all messages $m \in\{0,1\}^{\ell}$, the following distributions are computationally indistinguishable:

$$
\begin{array}{r}
\left\{\operatorname{rGen}\left(r_{\mathrm{G}}\right), \operatorname{rRndEnc}\left(r_{\mathrm{G}}, r_{\mathrm{E}}, m\right), \mathrm{PK}, c \mid(\mathrm{PK}, \mathrm{SK})=\operatorname{Gen}\left(1^{k} ; r_{\mathrm{G}}\right), c=\operatorname{Enc}_{\mathrm{PK}}\left(m ; r_{\mathrm{E}}\right)\right\} \\
\text { and }\left\{\hat{r}_{\mathrm{G}}, \hat{r}_{\mathrm{E}}, \hat{\mathrm{PK}}, \hat{c} \mid(\mathrm{PK}, \perp)=\mathrm{oGen}\left(1^{k} ; \hat{r}_{\mathrm{G}}\right), \hat{c}=\operatorname{oRndEnc}_{\mathrm{PK}}\left(1^{k} ; \hat{r}_{\mathrm{E}}\right)\right\}
\end{array}
$$

It follows from the definition that a trapdoor simulatable encryption scheme is also semantically secure.

\footnotetext{
${ }^{6}$ Existence of such inverting algorithms is called trapdoor invertibility. Compared to the simulatable cryptosystem (without trapdoor) defined in [DN00], rGen (resp. rRndEnc) takes $r_{\mathrm{G}}\left(\right.$ resp. $\left.\left(r_{\mathrm{G}}, r_{\mathrm{E}}, m\right)\right)$ as the additional trapdoor information.
} 
Encrypting longer messages. We note that if we started only with a trapdoor simulatable PKE for single bits, we may encrypt a longer message of length $n$ by generating a single public key PK using Gen, and concatenating each of the message encrypted under PK.

\section{Non-Committing Encryption from Weaker Assumptions}

Theorem 4. Suppose there exists a trapdoor simulatable encryption scheme. Then, there exists a non-committing encryption scheme as well as a universally composable oblivious transfer protocol secure against semi-honest, adaptive adversaries.

We show how to construct a non-committing bit encryption scheme (NCGen, NCEnc, NCDec, NCSim) from a $k$-bit trapdoor simulatable PKE (Gen, Enc, Dec) (augmented with (oGen, oRndEnc, rGen, rRndEnc)). This is sufficient to establish the theorem by the connection between encrypting single bits and multiple bits as discussed in Sections 3 and 4 Our construction is presented in Figures 2 and 3.

Correctness. We begin by establishing correctness.

- Assume that the input $\left[c_{1}, \ldots, c_{4 k}\right]$ to the decryption algorithm is a random encryption of 0 . Recall that $J=\left\{\operatorname{Dec}_{\mathrm{SK}_{i}}\left(c_{i}\right) \mid i \in T\right\}$ and we will output 0 unless $M_{0} \notin J$. It is easy to see that $\operatorname{Pr}\left[M_{0} \notin J\right] \leq\left(\begin{array}{c}3 k \\ k\end{array}\right) /\left(\begin{array}{c}4 k \\ k\end{array}\right)+2^{-\Omega(k)}$ where the first summand comes from the probability that $S \cap T=\emptyset$ and the second

Key Generation NCGen $\left(1^{k}\right)$ :

1. Pick $M_{0}, M_{1}$ at random from $\{0,1\}^{k}$.

2. Choose a random subset $T \subseteq[4 k]$ of size $k$.

3. For $i=1,2, \ldots, 4 k$, generate a pair $\left(\mathrm{PK}_{i}, \mathrm{SK}_{i}\right)$ as follows:

$$
\left(\mathrm{PK}_{i}, \mathrm{SK}_{i}\right)= \begin{cases}\mathrm{Gen}\left(1^{k}\right) & \text { if } i \in T \\ \mathrm{oGen}\left(1^{k}\right) & \text { otherwise }\end{cases}
$$

Set $e=\left[M_{0}, M_{1}, \mathrm{PK}_{1}, \ldots, \mathrm{PK}_{4 k}\right]$ and $d=\left[T, \mathrm{SK}_{1}, \ldots, \mathrm{SK}_{4 k}\right]$.

Encryption NCEnc $\mathrm{PK}_{\mathrm{PK}}(b)$ :

1. Choose a random subset $S \subseteq[4 k]$ of size $k$.

2. For $i=1,2, \ldots, 4 k$, generate a ciphertext $c_{i}$ as follows:

$$
c_{i}= \begin{cases}\operatorname{Enc}_{\mathrm{PK}_{i}}\left(M_{b}\right) & \text { if } i \in S \\ \operatorname{oRndEnc}_{\mathrm{PK}_{i}}\left(1^{k}\right) & \text { otherwise }\end{cases}
$$

Set $c=\left[c_{1}, \ldots, c_{4 k}\right]$.

Decryption $\operatorname{NCDec}_{\mathrm{PK}}(c)$ :

1. Compute $J=\left\{\operatorname{Dec}_{\mathrm{PK}_{i}}\left(c_{i}\right) \mid i \in T\right\}$.

2. If $M_{0} \in J$, output 0 ; else, output 1 .

Fig. 2. Non-Committing Encryption Scheme (NCGen, NCEnc, NCDec) 


\section{Simulation NCSim:}

1. Pick $M_{0}, M_{1}$ at random from $\{0,1\}^{k}$.

2. Picking the sets $S_{0}, S_{1}, T_{0}, T_{1}$ :

- Pick two random subsets $S_{0}, T_{0}$ of $[4 k]$ each of size $k$.

- Pick two random subsets $S_{1}, T_{1}$ of $[4 k] \backslash\left(S_{0} \cup T_{0}\right)$ such that $\left|S_{1} \cap T_{1}\right|=\left|S_{0} \cap T_{0}\right|$.

3. Generating the keys: for $i=1,2, \ldots, 4 k$, set

$$
\left(\mathrm{PK}_{i}, \mathrm{SK}_{i}\right)= \begin{cases}\mathrm{Gen}\left(1^{k} ; r_{\mathrm{G}}^{i}\right) & \text { if } i \in T_{0} \cup S_{0} \cup T_{1} \cup S_{1} \\ \mathrm{oGen}\left(1^{k} ; \hat{\mathrm{G}}_{\mathrm{G}}^{i}\right) & \text { otherwise }\end{cases}
$$

4. Generating the ciphertext: for $i=1,2, \ldots, 4 k$, set

$$
c_{i}= \begin{cases}\operatorname{Enc}_{\mathrm{PK}_{i}}\left(M_{0} ; r_{\mathrm{E}}^{i}\right) & \text { if } i \in S_{0} \\ \operatorname{Enc}_{\mathrm{PK}_{i}}\left(M_{1} ; r_{\mathrm{E}}^{i}\right) & \text { if } i \in S_{1} \\ \text { oRndEnc }_{\mathrm{PK}_{i}}\left(\hat{r}_{\mathrm{E}}^{i}\right) & \text { otherwise }\end{cases}
$$

5. Simulating an opening to $b$ : set $\sigma_{\mathrm{G}}^{b}=\left\{T_{b}, u_{\mathrm{G}}^{b, 1}, \ldots, u_{\mathrm{G}}^{b, 4 k}\right\}$ and $\sigma_{\mathrm{E}}^{b}=$ $\left\{S_{b}, u_{\mathrm{E}}^{b, 1}, \ldots, u_{\mathrm{E}}^{b, 4 k}\right\}$, where

$$
\begin{aligned}
& u_{\mathrm{G}}^{b, i}= \begin{cases}r_{\mathrm{G}}^{i} & \text { if } i \in T_{b} \\
\mathrm{rGen}\left(r_{\mathrm{G}}^{i}\right) & \text { if } i \in T_{0} \cup T_{1} \cup S_{0} \cup S_{1} \backslash T_{b} \\
\hat{r}_{\mathrm{G}}^{i} & \text { otherwise }\end{cases} \\
& u_{\mathrm{E}}^{b, i}= \begin{cases}r_{\mathrm{E}}^{i} & \text { if } i \in S_{b} \\
\operatorname{rRndEnc}\left(r_{\mathrm{G}}^{i}, r_{\mathrm{E}}^{i}, M_{1-b}\right) & \text { if } i \in S_{1-b} \\
\hat{r}_{\mathrm{E}}^{i} & \text { otherwise }\end{cases}
\end{aligned}
$$

Set $e=\left[M_{0}, M_{1}, \mathrm{PK}_{1}, \ldots, \mathrm{PK}_{4 k}\right], c=\left[c_{1}, \ldots, c_{4 k}\right]$. Additionally output $\sigma_{\mathrm{G}}^{0}, \sigma_{\mathrm{E}}^{0}, \sigma_{\mathrm{G}}^{1}, \sigma_{\mathrm{E}}^{1}$.

Fig. 3. Non-Committing Encryption Scheme NCSim

bounds the probability of a decryption error in the underlying encryption scheme (Gen, Enc, Dec).

- Assume that the input $\left[c_{1}, \ldots, c_{4 k}\right]$ to the decryption algorithm is a random encryption of 1 . Recall that $J=\left\{\operatorname{Dec}_{\mathrm{SK}_{i}}\left(c_{i}\right) \mid i \in T\right\}$ and we will output 1 unless $M_{0} \in J$. To bound $\operatorname{Pr}\left[M_{0} \in J\right]$, observe that the distribution of $J$ depends only on $M_{1}, \mathrm{PK}_{1}, \ldots, \mathrm{PK}_{4 k}, T, \mathrm{SK}_{1}, \ldots, \mathrm{SK}_{4 k}$ and the coin tosses used to generate $c_{1}, \ldots, c_{4 k}$, and is therefore independent of the choice of a random $M_{0}$. This means that for each $i \in T$, the probability that $\operatorname{Dec}_{\mathrm{SK}_{i}}\left(c_{i}\right)$ equals $M_{0}$ is $2^{-k}$. Taking a union bound, we obtain $\operatorname{Pr}\left[M_{0} \in J\right] \leq k \cdot 2^{-k}$.

Security. We need to show that for each $b=0,1$, a normal encryption of $b$ and a simulated encryption of $b$ are computationally indistinguishable. Note that the view in a normal encryption of $b$ contains two sets $T, S$ which we will label as $T_{b}, S_{b}$ and we will append to the view two sets $T_{1-b}, S_{1-b}$ that are determined as follows: pick two random subsets $S_{1-b}, T_{1-b}$ of $[4 k] \backslash\left(S_{b} \cup T_{b}\right)$ such that $\left|S_{1} \cap T_{1}\right|=\left|S_{0} \cap T_{0}\right|$; call this distribution 
$H_{0}$. We will also append to the view in a simulated encryption of $b$ the sets $T_{1-b}, S_{1-b}$ as determined by the experiment NCSim; call this distribution $H_{4 k}$. We will show that the augmented distributions $H_{0}$ and $H_{4 k}$ are computationally indistinguishable in two steps:

Reasoning about the sets. First, we claim that the 4-tuple $\left(S_{0}, T_{0}, S_{1}, T_{1}\right)$ in the augmented distribution $H_{0}$ and in $H_{4 k}$ are identically distributed. If $b=0$, this is obvious since the distributions are defined in exactly the same way. The case for $b=1$ follows from a symmetry argument, namely that if we switch $\left(S_{0}, T_{0}\right)$ with $\left(S_{1}, T_{1}\right)$ in the experiment NCSim, we get exactly the same distribution. Henceforth, it suffices to argue that $H_{0}$ and $H_{4 k}$ are computationally indistinguishable, conditioned on some fixed $\left(S_{0}, T_{0}, S_{1}, T_{1}\right)$ in both $H_{0}$ and $H_{4 k}$. We may now WLOG focus on the case $b=0$. In fact, we may as well also fix $M_{0}, M_{1}$ in both $H_{0}$ and $H_{4 k}$. In addition to $S_{0}, T_{0}, S_{1}, T_{1}, M_{0}, M_{1}$, the distributions $H_{0}, H_{4 k}$ comprise:

- $4 k$ public keys $\mathrm{PK}_{1}, \ldots, \mathrm{PK}_{4 k}$ (generated using either Gen or oGen);

- $4 k$ ciphertexts $c_{1}, \ldots, c_{4 k}$ (generated using either Enc or oRndEnc);

- $4 k$ sets of coin tosses $u_{\mathrm{G}}^{1}, \ldots, u_{\mathrm{G}}^{4 k}$ for generating the public/secret keys; and

- $4 k$ sets of coin tosses $u_{\mathrm{E}}^{1}, \ldots, u_{\mathrm{E}}^{4 k}$ for generating the ciphertexts.

That is, we have $4 k$ tuples of the form $\left(\mathrm{PK}_{i}, c_{i}, u_{\mathrm{G}}^{i}, u_{\mathrm{E}}^{i}\right), i=1, \ldots, 4 k$ in each view. Since $S_{0}, T_{0}, S_{1}, T_{1}$ are fixed, each of these $4 k$ tuples are independently sampled from some distribution that only depends on the index $i$. Denote by $X_{1}, \ldots, X_{4 k}$ the random variables for the $4 k$ tuples in $H_{0}$, and $Y_{1}, \ldots, Y_{4 k}$ the random variables for the $4 k$ tuples in $H_{4 k}$.

The hybrid argument. Next, we argue that $X_{i}$ and $Y_{i}$ are computationally indistinguishable for $i=1, \ldots, 4 k$, from which the indistinguishability of $H_{0}$ and $H_{4 k}$ follows via a hybrid argument. There are several cases we need to consider:

- $i \in T_{0}$ or $i \in[4 k] \backslash\left(T_{0} \cup S_{0} \cup T_{1} \cup S_{1}\right)$. It is easy to verify that in either of these cases, $X_{i}$ and $Y_{i}$ are identically distributed.

- $i \in S_{1}$ ("oGen, oRndEnc $\cong$ Gen, Enc"). Here, $X_{i}$ is the distribution

$$
\left\{\hat{\mathrm{PK}}, \hat{c}, \hat{r}_{\mathrm{G}}, \hat{r}_{\mathrm{E}} \mid(\hat{\mathrm{PK}}, \perp)=\mathrm{oGen}\left(\hat{r}_{\mathrm{G}}\right), \hat{c}=\mathrm{oRndEnc}_{\mathrm{PK}}\left(\hat{r}_{\mathrm{E}}\right)\right\}
$$

and $Y_{i}$ is the distribution

$$
\left\{\mathrm{PK}, c, \operatorname{rGen}\left(r_{\mathrm{G}}\right), \operatorname{rRndEnc}\left(r_{\mathrm{G}}, r_{\mathrm{E}}, M_{1}\right) \mid(\mathrm{PK}, \mathrm{SK})=\operatorname{Gen}\left(r_{\mathrm{G}}\right), c=\operatorname{Enc}_{\mathrm{PK}}\left(M_{1} ; r_{\mathrm{E}}\right)\right\} \text {. }
$$

Indistinguishability follows immediately from the security of the trapdoor simulatable PKE.

- $i \in S_{0} \backslash T_{0}$ ("oGen, Enc $\cong$ Gen, Enc"). Here, $X_{i}$ is the distribution

$$
\left\{\hat{\mathrm{PK}}, c, \hat{r}_{\mathrm{G}}, r_{\mathrm{E}} \mid(\hat{\mathrm{PK}}, \perp)=\mathrm{oGen}\left(\hat{r}_{\mathrm{G}}\right), c=\mathrm{Enc}_{\mathrm{PK}}\left(M_{0} ; r_{\mathrm{E}}\right)\right\}
$$

and $Y_{i}$ is the distribution

$$
\left\{\mathrm{PK}, c, \mathrm{rGen}\left(r_{\mathrm{G}}\right), r_{\mathrm{E}} \mid(\mathrm{PK}, \mathrm{SK})=\operatorname{Gen}\left(r_{\mathrm{G}}\right), c=\mathrm{Enc}_{\mathrm{PK}}\left(M_{0} ; r_{\mathrm{E}}\right)\right\} .
$$


Indistinguishability follows again from the security of the trapdoor simulatable PKE.

- $i \in T_{1} \backslash S_{1}$ ("oGen, oRndEnc $\cong$ Gen, oRndEnc"). Here, $X_{i}$ is the distribution

$$
\left\{\hat{\mathrm{PK}}, \hat{c}, \hat{r}_{\mathrm{G}}, \hat{r}_{\mathrm{E}} \mid(\hat{\mathrm{PK}}, \perp)=\mathrm{oGen}\left(\hat{r}_{\mathrm{G}}\right), \hat{c}=\mathrm{oRndEnc}_{\mathrm{PK}}\left(\hat{r}_{\mathrm{E}}\right)\right\}
$$

and $Y_{i}$ is the distribution

$$
\left\{\mathrm{PK}, \hat{c}, \operatorname{rGen}\left(r_{\mathrm{G}}\right), \hat{r}_{\mathrm{E}} \mid(\mathrm{PK}, \mathrm{SK})=\operatorname{Gen}\left(r_{\mathrm{G}}\right), \hat{c}=\operatorname{oRndEnc}_{\mathrm{PK}}\left(\hat{r}_{\mathrm{E}}\right)\right\} .
$$

Indistinguishability follows again from the security of the trapdoor simulatable PKE.

Improving the Efficiency. Instead of using sets $S, T \subset[4 k]$ of size $k$, we choose $S, T \subset[40]$ of size 10 . The previous analysis still goes through, except we now have a constant decryption error. To address this problem, we first encode the message 7 with a linear-rate error-correcting code that corrects a constant fraction of errors, and then encrypt the codeword with the encryption scheme with constant error.

\section{Trapdoor Simulatable PKE from Hardness of Factoring}

Theorem 5. Suppose factoring Blum integers is hard on average, and that Blum integers are dense, then there exists a trapdoor simulatable PKE.

For simplicity, we only present a 1-bit trapdoor simulatable encryption scheme; we may encrypt longer messages by encrypting bit by bit.

A number-theoretic lemma. Fix any $k$-bit integer modulus $N$ and we will work with the group $Z_{N}^{*}$. We will use factor $(N)$ to denote the factorization of $N$, and we define $Q_{N}=\left\{a^{2^{k}} \mid a \in Z_{N}^{*}\right\}$. Now, consider the map $\psi_{N}: Q_{N} \rightarrow Q_{N}$ given by $\psi_{N}(x)=$ $x^{2}(\bmod N)$. As shown in [H99, Facts 3.5-3.7], $\psi_{N}$ defines a permutation on $Q_{N}$. We provide a more direct proof which also yields an efficient algorithm to invert $\psi_{N}$ given factor $(N)$.

Claim. The map $\psi_{N}$ defines a permutation on $Q_{N}$.

Proof. Let $q$ denote the largest odd divisor of $\phi(N)$, where $\phi(\cdot)$ is the Euler's totient function. It is easy to see that $\phi(N)$ divides $2^{k} q$, since $N<2^{k}$. Take any $y \in Q_{N}$, where $y=a^{2^{k}}$. Then by Euler's theorem, $y^{q}=1(\bmod N)$ and thus $\psi_{N}\left(y^{(q+1) / 2}\right)=$ $y(\bmod N)$. Clearly, $y^{(q+1) / 2} \in Q_{N}$, so the map $\psi_{N}$ is surjective. Moreover, the range and domain of $\psi_{N}$ have equal sizes, so $\psi_{N}$ must define a bijection.

The construction. We sketch the construction here; the formal construction is shown in Figure 4.

\footnotetext{
${ }^{7}$ The codeword length (or, equivalently the message length) should be $\Omega(k)$. Then, by Chernoff bound, the number of decryption errors remains a constant fraction of the codeword length with overwhelming probability.
} 
Key generation Gen $\left(1^{k}\right)$ :

1. Run Bach's algorithm using the randomness $r_{\mathrm{G}}$ to sample random $N_{1}, \ldots, N_{k^{3}} \in$ $\{0,1\}^{k}$ along with their factorization factor $\left(N_{1}\right), \ldots$, factor $\left(N_{k^{3}}\right)$.

2. Set PK $=\left[N_{1}, \ldots, N_{k^{3}}\right]$ and SK $=\left[\right.$ factor $\left(N_{1}\right), \ldots$, factor $\left.\left(N_{k^{3}}\right)\right]$.

Encryption $\operatorname{Enc}(b)$ :

1. Parse the randomness $r_{\mathrm{E}}$ as $\left(a_{1}, \ldots, a_{k^{3}}\right) \in Z_{N_{1}}^{*} \times \cdots \times Z_{N_{k^{3}}}^{*}, r_{1}, \ldots, r_{k^{3}} \in\{0,1\}^{k}$ and $b_{1}, \ldots, b_{k^{3}-1} \in\{0,1\}$.

2. Compute $b_{k^{3}}=b \oplus b_{1} \oplus \cdots \oplus b_{k^{3}-1}$.

3. Compute $x_{i}=a_{i}^{2^{k}} \in Q_{N_{i}}, i=1, \ldots, k^{3}$.

4. Output $\left[\pi_{N_{i}}\left(x_{i}\right), r_{i},\left(x_{i} \cdot r_{i}\right) \oplus b_{i}, i=1, \ldots, k^{3}\right]$.

Decryption $\operatorname{Dec}(c)$ :

1. Parse $c$ as $\left[y_{i}, r_{i}, \beta_{i}, i=1, \ldots, k^{3}\right]$.

2. Compute $b_{i}=\left(\pi_{N_{i}}^{-1}\left(y_{i}\right) \cdot r_{i}\right) \oplus \beta_{i}, i=1, \ldots, k^{3}$.

3. Output $b_{1} \oplus \cdots \oplus b_{k^{3}}$.

Oblivious key generation oGen $\left(1^{k}\right)$ :

1. Parse the randomness $\hat{r}_{\mathrm{G}} \in\{0,1\}^{k^{4}}$ as $N_{1}, \ldots, N_{k^{3}} \in\{0,1\}^{k}$.

2. Output $\left(N_{1}, \ldots, N_{k^{3}}\right)$.

Trapdoor invertibility key generation $\mathrm{rGen}\left(r_{\mathrm{G}}\right)$ :

1. Run Gen $\left(r_{\mathrm{G}}\right)$ to obtain $\hat{r}_{\mathrm{G}}=\left(N_{1}, \ldots, N_{k^{3}}\right)$.

2. Output $\hat{r}_{\mathrm{G}}$.

Oblivious sampling of ciphertexts oRndEnc $\left(1^{k}\right)$ :

1. Parse the randomness $\hat{r}_{\mathrm{E}}$ as $\left(\gamma_{1}, \ldots, \gamma_{k^{3}}\right) \in Z_{N_{1}}^{*} \times \cdots \times Z_{N_{k^{3}}}^{*}, s_{1}, \ldots, s_{k^{3}} \in\{0,1\}^{k}$ and $\beta_{1}, \ldots, \beta_{k^{3}} \in\{0,1\}$.

2. Compute $y_{i}=\gamma_{i}^{2^{k}} \in Q_{N_{i}}, i=1, \ldots, k^{3}$.

3. Output $\left[y_{i}, s_{i}, \beta_{i}, i=1, \ldots, k^{3}\right]$.

Trapdoor invertibility for ciphertexts $r \operatorname{RndEnc}\left(r_{\mathrm{G}}, r_{\mathrm{E}}, b\right)$ :

1. Use $r_{\mathrm{G}}$ to compute factor $\left(N_{1}\right), \ldots$, factor $\left(N_{k}\right)$. and parse $r_{\mathrm{E}}$ as in Enc.

2. Set $s_{i}=r_{i}$ and $\beta_{i}=\left(x_{i} \cdot r_{i}\right) \oplus b_{i}, i=1, \ldots, k^{3}$.

3. Pick a random $\gamma_{i}$ uniformly from the set $\left\{\gamma_{i} \in Z_{N_{i}}^{*} \mid \gamma_{i}^{2^{k}}=\pi_{N_{i}}\left(x_{i}\right)\right\}$.

4. Output $\hat{r}_{\mathrm{E}}=\left(\gamma_{1}, \ldots, \gamma_{k^{3}}, s_{1}, \ldots, s_{k^{3}}, \beta_{1}, \ldots, \beta_{k^{3}}\right)$.

Fig. 4. Trapdoor Simulatable PKE from hardness of factoring Blum integers

STEP 1: First, we construct a family of "weakly one-way" enhanced trapdoor permutations. We start by modifying $\psi_{N}$ to obtain a new family of permutations $\pi_{N}$; the modification is analogous to that in [G04, Section C.1] to obtain enhanced trapdoor permutations from Rabin's trapdoor permutations. The permutations $\pi_{N}$ : $Q_{N} \rightarrow Q_{N}$ are indexed by a $k$-bit integer $N$ and is given by:

$$
\pi_{N}(x) \stackrel{\text { def }}{=} \psi_{N}^{k+1}(x)=x^{2^{k+1}} \quad(\bmod N)
$$

and the trapdoor is factor $(N)$. We may sample from this family by running Bach's algorithm [B88, K02] to pick a random $k$-bit integer along with its factorization.

It is easy to verify $\pi_{N}$ is a family of trapdoor permutations. Clearly, $\pi_{N}$ is a permutation because it is the $(k+1)$-fold iterate of a permutation $\psi_{N}$. Given the index $N, \pi_{N}$ is efficiently computable by repeated squaring. Given the trapdoor factor $(N), \pi_{N}^{-1}$ is efficiently computable given factor $(N)$, by simply mapping $y$ 
to $y^{((q+1) / 2)^{k+1}}$, i.e., raising $y$ to the $(q+1) / 2$ 'th power $k+1$ times. Here, $q$ denotes the largest odd divisor of $\phi(N)$, which is easy to compute with the trapdoor. Moreover, we can show that if $N$ is a Blum integer (which occurs with probability $\Omega\left(1 / k^{2}\right)$ GM04, RS94), then inverting $\pi_{N}$ given $N$ is at least as hard as factoring $N$. This implies that $\pi_{N}$ is one-way with probability $\Omega\left(1 / k^{2}\right)$ over the choice of $N$. STEP 2: Construct a "weak" encryption scheme using the standard construction of PKE from trapdoor permutations via the Goldreich-Levin hard-core predicate. The public key is $N$, the secret key is factor $(N)$, and to encrypt a bit $b$, we pick a random $x \in Q_{N}, r \in\{0,1\}^{k}$ and output $\left(\pi_{N}(x), r,(x \cdot r) \oplus b\right)$, where $x \cdot r$ is the standard dot-product of $k$-bit strings. Again, this scheme will be semantically secure with probability $\Omega\left(1 / k^{2}\right)$ over the choice of $N$.

STEP 3: To boost the security of the "weak" encryption scheme, we define a new scheme where the public key is $k^{3}$ random $k$-bit strings $N_{1}, \ldots, N_{k^{3}}$ (with overwhelming probability, one of these is a Blum integer), and to encrypt a bit $b$, we pick random $b_{1}, \ldots, b_{k^{3}}$ such that $b=b_{1} \oplus \cdots b_{k^{3}}$ and concatenate the encryptions of $b_{1}, \ldots, b_{k^{3}}$ under the respective public keys $N_{1}, \ldots, N_{k^{3}}$. By a standard argument (c.f. [Y82, DP92]), this encryption scheme is semantically secure in the standard sense.

Analysis. Indeed, we claim something stronger - that the encryption scheme derived in Step 3 is a trapdoor simulatable PKE.

- (Oblivious sampling \& trapdoor invertibility for key generation) This is trivial, since a random public key corresponds to a string in $\{0,1\}^{4 k}$. We can clearly sample such a public key without learning the secret key.

- (Oblivious sampling \& trapdoor invertibility for random ciphertext) For simplicity, we present the algorithms for sampling random ciphertext for the scheme obtained in Step 2. Here, sampling is easy: on input the public key $N$, pick $\gamma \in Z_{N}^{*}, s \in$ $\left.\{0,1\}^{k}, \beta \in\{0,1\}\right)$ and output $\left(\gamma^{2^{k}}, s, \beta\right)$. To implement reverse sampling, we need an efficient algorithm that given factor $(N)$ and $x \in Q_{N}$, output a random element of the set $\left\{\gamma \in Z_{N}^{*} \mid \gamma^{2^{k}}=\pi_{N}(x)=x^{2^{k+1}}\right\}$. This can be accomplished as follows: pick a random $\eta \in Z_{N}^{*}$ and output $x^{2} \cdot \eta /\left(\eta^{2^{k}}\right)^{((q+1) / 2)^{k}}$, where $q$ is as before the largest odd divisor of $\phi(N)$. This works because $\eta /\left(\eta^{2^{k}}\right)^{((q+1) / 2)^{k}}$ will be a random $2^{k}$ 'th root of $1(\bmod N)$.

For the actual proof of security, we will need to show that if $N$ is a random Blum integer, then the following distributions are computationally indistinguishable for every $b$ :

$$
\left\{\left(N, \gamma, \pi_{N}(x), r,(x \cdot r) \oplus b\right)\right\} \text { and }\left\{\left(N, \gamma, \gamma^{2^{k}}, r, \beta\right)\right\}
$$

The first distribution corresponds to an encryption of $b$ using modulus $N$ and randomness $(x, r)$ along with $\gamma$ the output of rRndEnc (a random solution to the equation $\left.\gamma^{2^{k}}=\pi_{N}(x)\right)$. The second corresponds to an obliviously generated ciphertext along with the randomness. If there exists an efficient distinguisher, then there exists an efficient procedure $A$ that on input $N, \gamma$, outputs $\pi_{N}^{-1}\left(\gamma^{2^{k}}\right)$ with noticeable probability. Since squaring is a bijection on quadratic residues modulo Blum integers, the output of $A$ is also the 4 th root of $\gamma^{2}$. We may then use a reduction in [G04, Section C.1] to derive from $A$ an algorithm for factoring $N$ with noticeable probability. 


\section{Oblivious Transfer and MPC}

We describe the construction underlying Theorem 3 which proceeds in two steps:

STEP 1: We begin with the [CLOS02] construction of a semi-honest OT protocol as applied to our non-committing encryption scheme, and observe that the protocol is secure against malicious senders. For that, we just need to show how to extract the sender's input when the receiver is honest. In this case, the simulator will generate the public keys sent by the receiver in the first message along with the secret keys, so that it can then extract the malicious sender's input by decrypting.

STEP 2: Next, we apply the compiler in CDSMW09] to "boost" the security guarantee from tolerating semi-honest receivers to tolerating malicious receivers. (Note that we will not need to apply OT reversal as in [CDSMW09.)

Acknowledgements. We thank Ran Canetti, Yuval Ishai, Jonathan Katz, and Chris Peikert for helpful discussions and clarifications.

\section{References}

[B88] Bach, E.: How to generate factored random numbers. SIAM J. Comput. 17(2), 179193 (1988)

[в97] Beaver, D.: Plug and play encryption. In: Kaliski Jr., B.S. (ed.) CRYPTO 1997. LNCS, vol. 1294, pp. 75-89. Springer, Heidelberg (1997)

[B98] Beaver, D.: Adaptively secure oblivious transfer. In: Ohta, K., Pei, D. (eds.) ASIACRYPT 1998. LNCS, vol. 1514, pp. 300-314. Springer, Heidelberg (1998)

[BH92] Beaver, D., Haber, S.: Cryptographic protocols provably secure against dynamic adversaries. In: Rueppel, R.A. (ed.) EUROCRYPT 1992. LNCS, vol. 658, pp. 307323. Springer, Heidelberg (1993)

[C00] Canetti, R.: Security and composition of multiparty cryptographic protocols. J. Cryptology 13(1), 143-202 (2000)

[CDSmw09] Choi, S.G., Dachman-Soled, D., Malkin, T., Wee, H.: Simple, black-box constructions of adaptively secure protocols. In: Reingold, O. (ed.) TCC 2009. LNCS, vol. 5444, pp. 387-402. Springer, Heidelberg (2009)

[CFGN96] Canetti, R., Feige, U., Goldreich, O., Naor, M.: Adaptively secure multi-party computation. In: STOC, pp. 639-648 (1996), Longer version http://www.wisdom.weizmann.ac.il/ naor/PAPERS/nce_abs.html

[CHк05] Canetti, R., Halevi, S., Katz, J.: Adaptively-secure, non-interactive public-key encryption. In: Kilian, J. (ed.) TCC 2005. LNCS, vol. 3378, pp. 150-168. Springer, Heidelberg (2005)

[CLOS02] Canetti, R., Lindell, Y., Ostrovsky, R., Sahai, A.: Universally composable two-party and multi-party secure computation. In: STOC, pp. 494-503 (2002)

[DN00] Damgård, I.B., Nielsen, J.B.: Improved non-committing encryption schemes based on a general complexity assumption. In: Bellare, M. (ed.) CRYPTO 2000. LNCS, vol. 1880, pp. 432-450. Springer, Heidelberg (2000)

[DP92] De Santis, A., Persiano, G.: Zero-knowledge proofs of knowledge without interaction. In: FOCS, pp. 427-436 (1992)

[FF02] Fischlin, M., Fischlin, R.: The representation problem based on factoring. In: Preneel, B. (ed.) CT-RSA 2002. LNCS, vol. 2271, pp. 96-113. Springer, Heidelberg (2002) 
[G04] Goldreich, O.: Foundations of Cryptography: Basic Applications, vol. II. Cambridge University Press, Cambridge (2004)

[GM84] Goldwasser, S., Micali, S.: Probabilistic encryption. J. Comput. Syst. Sci. 28(2), 270-299 (1984)

[GM04] Granville, A., Martin, G.: Prime number races (2004), http://arxiv.org/abs/math/0408319

[GPV08] Gentry, C., Peikert, C., Vaikuntanathan, V.: Trapdoors for hard lattices and new cryptographic constructions. In: STOC, pp. 197-206 (2008)

[GWZ09] Garay, J.A., Wichs, D., Zhou, H.-S.: Somewhat non-committing encryption and efficient adaptively secure oblivious transfer. In: Halevi, S. (ed.) CRYPTO 2009. LNCS, vol. 5677, pp. 505-523. Springer, Heidelberg (2009)

[H99] Halevi, S.: Efficient commitment schemes with bounded sender and unbounded receiver. J. Cryptology 12(2), 77-89 (1999)

[IPS08] Ishai, Y., Prabhakaran, M., Sahai, A.: Founding cryptography on oblivious transfer - efficiently. In: Wagner, D. (ed.) CRYPTO 2008. LNCS, vol. 5157, pp. 572-591. Springer, Heidelberg (2008)

[JL00] Jarecki, S., Lysyanskaya, A.: Adaptively secure threshold cryptography: Introducing concurrency, removing erasures (Extended abstract). In: Preneel, B. (ed.) EUROCRYPT 2000. LNCS, vol. 1807, pp. 221-242. Springer, Heidelberg (2000)

[K02] Kalai, A.: Generating random factored numbers, easily. In: SODA, pp. 412-412 (2002)

[KO04] Katz, J., Ostrovsky, R.: Round-optimal secure two-party computation. In: Franklin, M. (ed.) CRYPTO 2004. LNCS, vol. 3152, pp. 335-354. Springer, Heidelberg (2004)

[PVw08] Peikert, C., Vaikuntanathan, V., Waters, B.: A framework for efficient and composable oblivious transfer. In: Wagner, D. (ed.) CRYPTO 2008. LNCS, vol. 5157, pp. 554-571. Springer, Heidelberg (2008)

[RS94] Rubinstein, M., Sarnak, P.: Chebyshevs bias. Experiment. Math 3(3), 173-197 (1994)

[S96] Schnorr, C.-P.: Security of $2^{t}$-root identification and signatures. In: Koblitz, N. (ed.) CRYPTO 1996. LNCS, vol. 1109, pp. 143-157. Springer, Heidelberg (1996)

[Y82] Yao, A.C.-C.: Theory and applications of trapdoor functions. In: FOCS, pp. 80-91 (1982) 